\title{
Paediatric cardiac CT examinations: impact of the iterative reconstruction method ASIR on image quality - preliminary findings
}

\author{
Frédéric A. Miéville • François Gudinchet • \\ Elena Rizzo • Phalla Ou • Francis Brunelle • \\ François O. Bochud • Francis R. Verdun
}

Received: 20 July 2010 /Revised: 26 January 2011 / Accepted: 15 February 2011 /Published online: 30 June 2011

(C) Springer-Verlag 2011

\begin{abstract}
Background Radiation dose exposure is of particular concern in children due to the possible harmful effects of ionizing radiation. The adaptive statistical iterative reconstruction (ASIR) method is a promising new technique that reduces image noise and produces better overall image quality compared with routine-dose contrast-enhanced methods. Objective To assess the benefits of ASIR on the diagnostic image quality in paediatric cardiac CT examinations.

Materials and methods Four paediatric radiologists based at two major hospitals evaluated ten low-dose paediatric cardiac examinations $\left(80 \mathrm{kVp}, \mathrm{CTDI}_{\mathrm{vol}}\right.$ 4.8-7.9 $\mathrm{mGy}$, DLP 37.1$178.9 \mathrm{mGy} \cdot \mathrm{cm}$ ). The average age of the cohort studied was 2.6 years (range 1 day to 7 years). Acquisitions were performed on a 64-MDCT scanner. All images were reconstructed at various ASIR percentages $(0-100 \%)$. For each examination, radiologists scored 19 anatomical structures
\end{abstract}

F. A. Miéville · F. O. Bochud •F. R. Verdun

Institute of Radiation Physics,

University Hospital Center and University of Lausanne,

Lausanne, Switzerland

F. Gudinchet $\cdot$ E. Rizzo

Department of Radiology,

University Hospital Center and University of Lausanne,

Lausanne, Switzerland

P. Ou $\cdot$ F. Brunelle

Department of Radiology, Necker Children's Hospital,

Paris, France

F. A. Miéville ( $\triangle)$

Institute of Radiation Physics - Medical Radiology,

University Hospital Center and University of Lausanne,

Grand-Pré 1,

Lausanne 1007, Switzerland

e-mail: frederic.mieville@chuv.ch using the relative visual grading analysis method. To estimate the potential for dose reduction, acquisitions were also performed on a Catphan phantom and a paediatric phantom. Results The best image quality for all clinical images was obtained with $20 \%$ and $40 \%$ ASIR $(p<0.001)$ whereas with ASIR above $50 \%$, image quality significantly decreased ( $p<$ 0.001). With $100 \%$ ASIR, a strong noise-free appearance of the structures reduced image conspicuity. A potential for dose reduction of about $36 \%$ is predicted for a 2- to 3-year-old child when using $40 \%$ ASIR rather than the standard filtered back-projection method.

Conclusion Reconstruction including $20 \%$ to $40 \%$ ASIR slightly improved the conspicuity of various paediatric cardiac structures in newborns and children with respect to conventional reconstruction (filtered back-projection) alone.

Keywords Adaptive statistical iterative reconstruction . Iterative reconstruction - Paediatric cardiac CT . Visual grading analysis

\section{Introduction}

Radiation dose exposure is of particular concern in children due to the possible harmful effects of ionizing radiation. In contrast to adults, neonates and children are more radiosensitive and have a longer lifetime to develop potential radiation injuries [1]. In a recent paper, Brenner and Hall predicted that up to $2 \%$ of future cancers in the USA may be related to prior CT examinations [2] and even if such projections are questionable, radiation protection of children during CT examinations should have high priority. Unfortunately, although the paediatric cardiology community has been constantly searching for less- 
harmful technology, multidetector CT remains the only imaging technology for very young children in whom MRI is not indicated or possible.

Over the last decade, CT scanner manufacturers have developed techniques to reduce patient exposure and to improve image quality, introducing automated tube current modulation or dual-source CT combined with high pitch values, for example [3-5]. Recently, the largest CT manufacturers have proposed new image reconstruction methods based on iterative reconstruction techniques. General Electric (GE) has launched the adaptive statistical iterative reconstruction (ASIR) method that reduces image noise and produces better overall image quality compared with routine-dose contrast-enhanced methods [6]. In practice, a combination of ASIR and the conventional filtered back-projection (FBP) reconstruction method can be chosen.

Recent studies have shown that this new reconstruction method is a promising technique that provides adequate diagnostic image quality at lower tube currents, leading to a reduction in patient dose [6]. Following a preliminary study, Hara et al. reported dose reductions of $32 \%-65 \%$ with $40 \%$ ASIR applied to routine abdomen or abdomen/ pelvis examinations in adults [7]. Marin et al. showed that $50 \%$ ASIR also had the potential to improve image quality and reduce the radiation dose in adult abdominal examinations at low tube voltages [8]. Recently, Leipsic et al. reported that the quality of images in adult cardiac CT angiography reconstructed using $40 \%$ or $60 \%$ ASIR was significantly improved [9]. ASIR at $50 \%$ is usually considered the best trade-off between dose reduction and diagnostic image quality. ASIR at $100 \%$ has been reported to introduce an appearance that is too noise-free leading to an artificial over-smoothing of the images [7]. Image oversmoothing with $100 \%$ ASIR has also been reported by Silva et al. although its diagnostic significance remains uncertain [6].

To the best of our knowledge, no investigation has been carried out to determine the potential benefits of using ASIR to improve the diagnostic image quality in paediatric cardiac examinations performed at low tube voltage that often produce images with motion artefacts due to high heart rates (up to $140 \mathrm{bpm}$ ) or patient movement. Therefore, an assessment of the impact of the ASIR approach in such examinations seems highly necessary.

The purpose of our study was to determine the benefit of ASIR on image quality in paediatric cardiac CT examinations acquired under low-dose conditions. Four radiologists working in two major medical centres evaluated the visibility of 19 anatomical structures reconstructed with various percentages of ASIR. The potential for dose reduction in paediatric cardiac examinations was also evaluated using a dedicated 3-D low-contrast paediatric phantom.

\section{Materials and methods}

Iterative reconstruction method: ASIR

ASIR is a reconstruction technique that is able to reduce image noise and improve image quality with respect to the traditional FBP method [6]. As described by Thibault et al. [10], the technique is based on a cost function, typically nonlinear, that optimizes the trade-offs between noise suppression and edge preservation. The process is repeated in successive iterative steps until the final estimated and ideal pixel values ultimately converge [11]. However, it has also been shown that the intrinsic characteristics of ASIR produce a noise-free reconstructed image with an unusual homogeneous appearance [6,7]. To restore the more classical appearance of CT images, a linear blend of the conventional FBP method with the ASIR has been implemented on GE CT consoles. Practically, the reconstructed image can be obtained with a blend of $10 \%$ to $100 \%$ ASIR, $0 \%$ corresponding to a conventional FBP image and $100 \%$ corresponding to a pure ASIR image.

\section{Study population}

The study cohort comprised ten children (six boys and four girls) requiring a cardiac $\mathrm{CT}$ examination because of chest pain and/or cardiac congenital malformations (tetralogy of Fallot, aortic valve stenosis, etc., or follow-up after Kawasaki disease). The average age of the population was 2.6 years (range 1 day to 7 years) and the median 3 years, and the average weight was $10.4 \mathrm{~kg}$ (range 2.4-20.0 kg). The thorax morphology was evaluated at the heart level from scout acquisitions. The average width and height of the thorax were $15.3 \mathrm{~cm}$ (range 8-19 cm) and $11.9 \mathrm{~cm}$ (range 7-15 cm), respectively. A beta-blocker (propranolol 10-20 mg according to the weight; Avlocardyl, AstraZeneca, Paris, France) was administrated orally $60-90 \mathrm{~min}$ before the CT scan in those with a heart rate higher than $75 \mathrm{bpm}$. Four patients were sedated using pentobarbital (Thiopental; Abbott Laboratories, North Chicago, IL) or chloral hydrate (Nervifene; Interdelta, Givisiez, Switzerland) orally or rectally $30 \mathrm{~min}$ before the procedure. No general anaesthesia was required. The institutional and ethical review boards of the participating hospitals approved the study protocol. Parents were informed, but no written consent was necessary as the $\mathrm{CT}$ examinations were indicated for clinical reasons. All the $\mathrm{CT}$ raw data and $\mathrm{CT}$ data files were anonymized. 


\section{Cardiac MDCT scanning}

Each patient underwent an ungated paediatric cardiac CT scan. All acquisitions were performed on a 64-MDCT scanner (LightSpeed VCT; GE Healthcare, Waukesha, WI) at an X-ray tube peak voltage of $80 \mathrm{kVp}$. The cardiac protocol was adapted to the patient's weight by adjusting the tube current limits (weight-adapted protocol used in clinical routine). During the CT acquisition, the tube current was automatically regulated by the automatic current modulation system with an upper limit fixed at $400 \mathrm{~mA}$, and the following parameters were maintained constant: detector configuration $(64 \times 0.625)$, scan field of view (paediatric body), noise index (NI, 15.5), tube current range associated with NI (159-400 mA), pitch value (0.984), gantry rotation time $(0.4 \mathrm{~s})$, reconstruction slice thickness $(0.625 \mathrm{~mm})$, and reconstruction convolution kernels (standard and lung). The scan was performed after intravenous injection of contrast medium (Omnipaque 300; GE Healthcare, Princeton, N.J.) at $2 \mathrm{ml} / \mathrm{kg}$. The indication to start CT acquisition was based on the bolus-tracking technique placed at the level of the ascending aorta. Images were acquired in inspiratory breath-hold when possible. For the cohort studied, the average volume CT dose index $\left(\mathrm{CTDI}_{\mathrm{vol}}\right)$ and doselength product were $6.7 \mathrm{mGy}$ (range 4.8-7.9 $\mathrm{mGy}$ ) and $112 \mathrm{mGy} \cdot \mathrm{cm}$ (range $37.1-178.9 \mathrm{mGy} \cdot \mathrm{cm}$ ), respectively. These values obtained for these paediatric cardiac CT scans were slightly above the diagnostic reference level recommended for a paediatric chest CT scan by the Swiss Federal Office of Public Health (the recommended $\mathrm{CTDI}_{\mathrm{vol}}$ values for thorax scans are $2,3.5,5.5$ and $8.5 \mathrm{mGy}$ in newborns, and in children ages 0 1, 2-5 and 6-10 years, respectively) [12], but were much lower than in previous European survey studies [13].
Phantom study

To study the effect of ASIR on the visibility of simple lowcontrast structures, we used a paediatric phantom comprising a 3-D low-contrast module (QRM-3DLC; QRM, Moehrendorf, Germany) surrounded by a soft tissueequivalent ring to mimic the X-ray attenuation of a 2- to 3 -year-old child (the length of the phantom is $10 \mathrm{~cm}$, the major axis $16 \mathrm{~cm}$ and the minor axis $12 \mathrm{~cm}$, as shown in Fig. 1a). The 3-D low-contrast module contains a cylindrical structure (20 $\mathrm{mm}$ in diameter) that produces a low contrast relative to the background of about $-10 \mathrm{HU}$. The behaviour of ASIR on high-contrast structure acquisitions was also evaluated using a quality control phantom (Catphan 600; Phantom Laboratory, Salem, N.Y.) as illustrated in Fig. 2b. Both phantoms were scanned using the paediatric cardiac $\mathrm{CT}$ protocol varying the tube current to get $\mathrm{CTDI}_{\mathrm{vol}}$ values in the range $1-8 \mathrm{mGy}$.

\section{Measurement of the radiation dose}

The accuracy of the $\mathrm{CTDI}_{\mathrm{vol}}$ displayed by the CT unit was verified by measuring the $\mathrm{CTDI}_{\mathrm{vol}}$ in a CTDI phantom of diameter $16 \mathrm{~cm}$ with a $10-\mathrm{cm}$ long CT pencil ion chamber connected to an electrometer (Radcal 103510.3 CTDI chamber, MDH 1015 electrometer; Radcal, Monrovia, CA). The ion chamber and electrometer were calibrated in RQR9 and RQA9 beams according to IEC 61267 [14], and traceable to the Swiss Federal Office of Metrology. For each set of acquisition parameters, the $\mathrm{CTDI}_{\mathrm{vol}}$ was calculated by dividing the weighted CTDI $\left(\mathrm{CTDI}_{\mathrm{w}}\right)$ by the pitch value, according to its definition $[15,16]$.

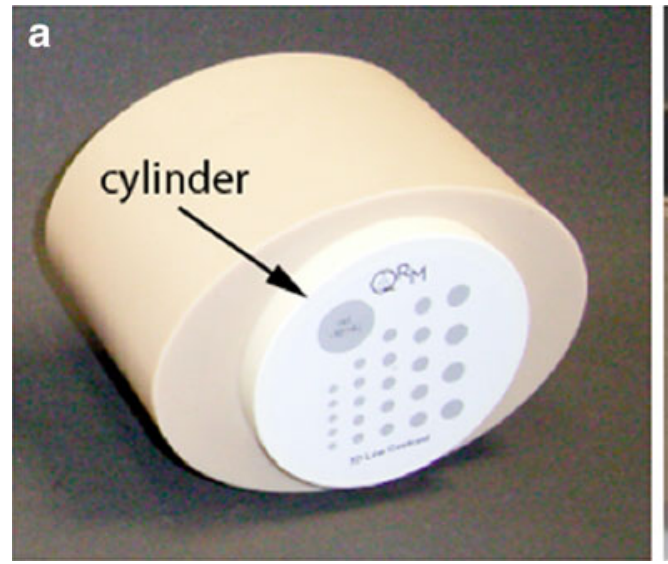

Fig. 1 Phantoms used in this study. a Paediatric phantom comprising a 3-D low-contrast module surrounded by a tissue-equivalent ring. The largest insert (arrow) is a cylinder $(20 \mathrm{~mm}$ in diameter, $25 \mathrm{~mm}$ in length) that produces a contrast of $-10 \mathrm{HU}$ relative to the background.

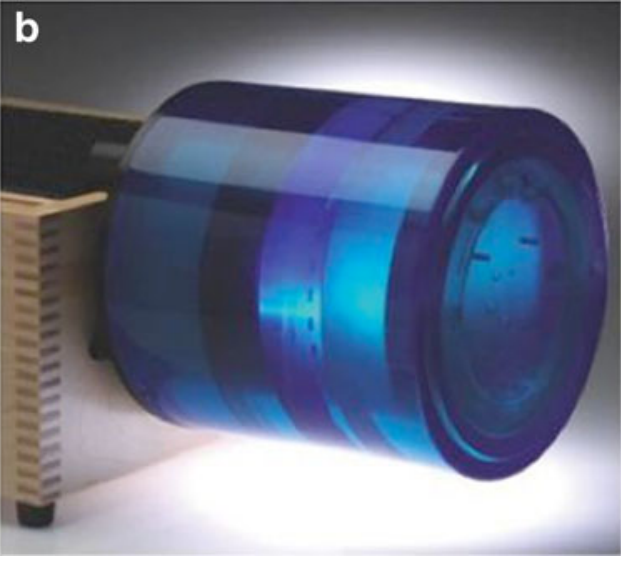

The length of the phantom is $10 \mathrm{~cm}$, the major axis $16 \mathrm{~cm}$ and the minor axis $12 \mathrm{~cm}$. b Catphan 600 phantom (200 $\mathrm{mm}$ in diameter) without a soft tissue-equivalent ring 

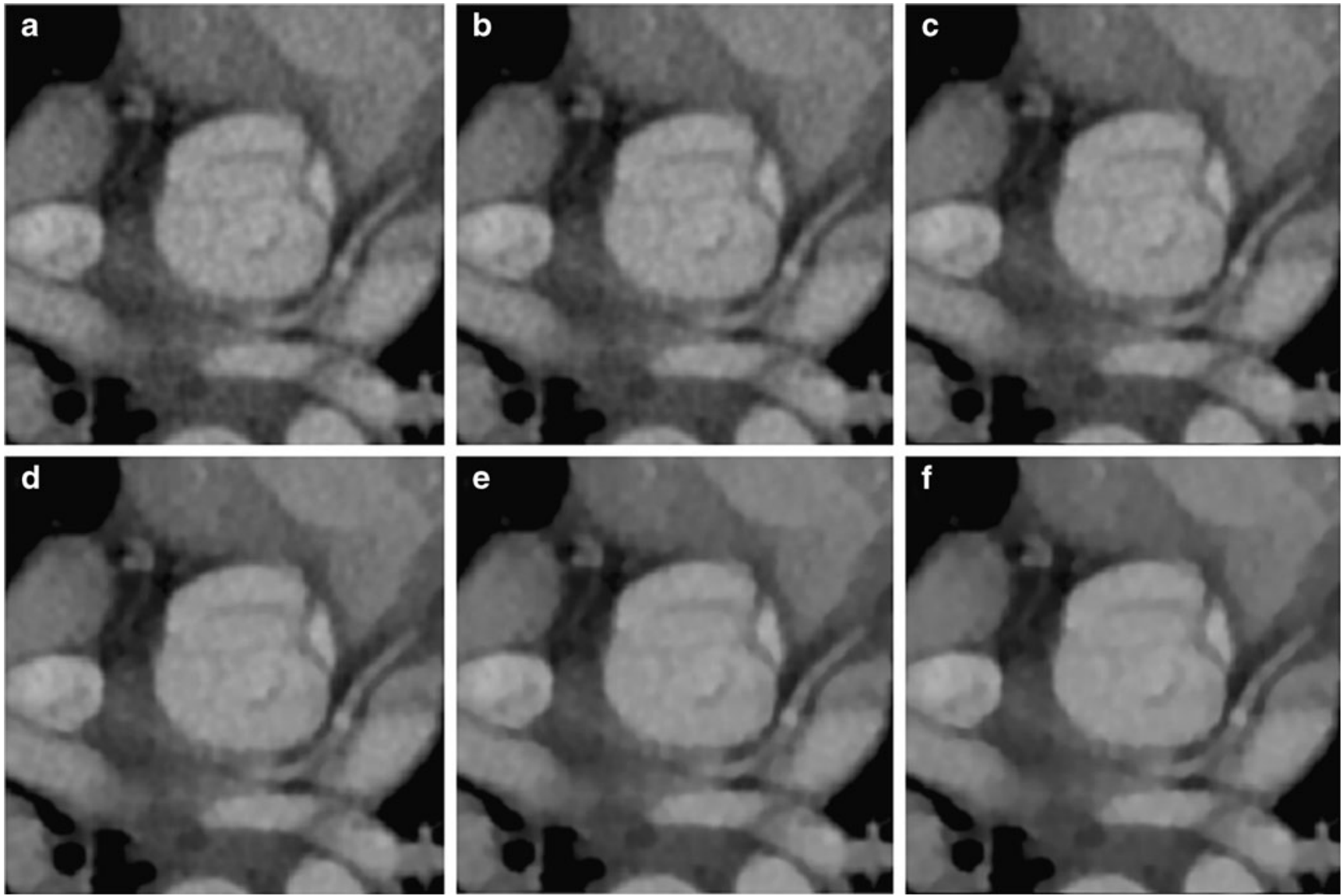

Fig. 2 Effects of the different percentages of ASIR on the visibility of the left coronary artery in a 3-year-old child. Images were reconstructed using the standard convolution kernel with: (a) the FBP method ( $0 \%$ ASIR), and (b-f) with $20 \%, 40 \%, 60 \%, 80 \%$ and $100 \%$

\section{Image reconstruction}

A commercial CT console (GE Healthcare, Waukesha, WI) designed for ASIR was used in the reconstructions of the images from the raw data for different ASIR percentages $(0 \%, 20 \%, 30 \%, 40 \%, 50 \%, 60 \%, 80 \%$ and $100 \%)$. It is worth mentioning that the reconstruction time with ASIR was not longer than with the FBP method. Since our cardiac paediatric CT scans are only reconstructed with the standard and lung reconstruction kernels, bone or soft tissue kernels were not assessed in this study. All patient images were reconstructed with a display field of view adapted to the patient's morphology, whereas the Catphan 600 phantom and the paediatric phantom images were displayed with a display field of view of $220 \mathrm{~mm}$ and $180 \mathrm{~mm}$, respectively. A fixed reconstruction matrix of $512 \times 512$ pixels was systematically used. The CT data files (DICOM files) were transferred from the CT unit to a standard desktop workstation where the images were analysed. Pixel-to-pixel subtraction (between images reconstructed at various ASIR percentage levels) was performed and the standard deviations (SD) were calculated
ASIR, respectively. The acquisition was carried out with the paediatric cardiac protocol at $80 \mathrm{kVp}$ and $6.33 \mathrm{mGy}$. Note the reduced conspicuity of the left coronary artery with $100 \%$ ASIR (f) compared with low percentages of ASIR (b and $\mathbf{c}$ )

using an in-house program written in MATLAB 7.7 (Mathworks, USA). Pixel-to-pixel image subtraction is the mathematical operation that takes two images as input and produces as output a third image whose pixel values are those of the first image minus the corresponding pixel values of the second image. The methodology used has been described in detail by Miéville et al. [17].

\section{Evaluation of clinical image quality}

Since the main objective of the study was to assess the effect of ASIR on the quality of images obtained at a standard dose in a small cohort of patients, no dose variation protocols were implemented. Image quality was thus evaluated at a given $\mathrm{CTDI}_{\mathrm{vol}}$ and assessed for diagnostic quality using relative visual grading analysis (VGA) [18].

The VGA method is considered to be closely related to the clinical task of assessing whether an abnormality is present in a medical image. This analysis was performed using the scan reconstructed with the standard FBP method 
Table 1 Set of structures established as diagnostic requirements to assess the diagnostic quality of paediatric cardiac images. The cardiac and thoracic structures were required to be sharp with clear visualization

\begin{tabular}{ll}
\hline Organ & Structure \\
\hline Left/right coronary artery & Ostium \\
& At $1.5 \mathrm{~cm}$ \\
& Distality \\
Cardiac cavity & Septum \\
& Left ventricle lateral wall \\
& Right ventricle lateral wall \\
& Aortic root \\
Aorta & Aortic cross \\
& Ascending aorta \\
& Descending aorta \\
& Pulmonary trunk \\
Pulmonary artery & Left pulmonary artery \\
& Right pulmonary artery \\
& Left pulmonary distality \\
& Right pulmonary distality \\
& Interstitium \\
\hline
\end{tabular}

${ }^{\mathrm{a}}$ The only structure reconstructed with the lung convolution kernel

as a reference against the reconstructions various ASIR percentages $(20 \%, 40 \%, 50 \%, 60 \%, 80 \%$ and $100 \%)$. A team of four paediatric radiologists (one junior with less than 10 years of experience, one experienced with less than 20 years of experience, and two senior with more than 20 years of experience) established a set of anatomical structures to evaluate the quality of the images using the same methodology as that published by the European Commission [19]. The list of anatomical structures proposed to grade image quality was accepted by all the radiologists involved in the study in spite of the fact that they did not work in the same hospital. Table 1 presents the 19 anatomical criteria (cardiac and thoracic structures) used in this study. All the structures where reconstructed with the standard convolution kernel except the lung interstitium for which the lung kernel was used. The VGA system used to grade the 19 selected structures is shown in Table 2. Each radiologist performed 1,140 comparisons (10 patients $\times 6$ ASIR percentages $\times 19$ structures). To minimize the potential biases of the study, the six ASIR reconstructions of each scan were randomized and the ASIR percentages were unknown to the radiologists. All the observers performed their analysis, independently, but on the same Advantage Windows workstation (GE Healthcare, Waukesha, WI) in a quiet room with low ambient lighting. The window level and window width could be freely adjusted for each reference structure but were then kept fixed to assess the associated ASIR-based reconstructed structures.
Statistical analysis

Mean differences in VGA scores between ASIR categories were evaluated using a repeated-measures analysis of variance (ANOVA) procedure (normality assumption, $p=$ 0.063 , obtained with the Shapiro-Wilk test), with a Bonferroni post hoc comparison to control for multiple pair-wise comparisons. One procedure was performed for each cardiac structure (19 separate procedures). Statistical significance was accepted at the $95 \%$ confidence level $(p<$ 0.05 ) for all repeated measures and analysis. Relative rating values were expressed as means \pm standard error of the mean (normal distribution assumption). All statistical analyses were performed using the commercial SPSS software (version 15.0; SPSS, Chicago, IL).

\section{Results}

Patient study

Figure 2 illustrates the effect of increasing the ASIR percentage from $0 \%$ to $100 \%$ in increments of $20 \%$. As expected, image noise was significantly reduced when the ASIR percentage increased. SD measurements in a region of interest located in the ascending aorta indicated a noise reduction of $58 \pm 5 \%$ between a standard FBP reconstruction ( $0 \%$ ASIR) and $100 \%$ ASIR. However, for high ASIR percentages, the image conspicuity was also modified because of the noise-free appearance.

Figure 3 shows the overall relative VGA scores as a function of ASIR percentage. The horizontal line at score zero indicates the limit between a score indicating improved visibility (score $>0$ ) and a score indicating reduced visibility (score $<0$ ) of the structures in comparison to the standard FBP (reference) images. The trend line in Fig. 3 indicates the average of the VGA scores obtained for each ASIR percentage.

Table 2 VGA scores used to grade the structure visibility

\begin{tabular}{ll}
\hline Relative score & $\begin{array}{l}\text { Visibility of the structure in relation } \\
\text { to the reference structure }\end{array}$ \\
\hline+3 & Definitely better \\
+2 & Better \\
+1 & Slightly better \\
0 & Equal \\
-1 & Slightly lower \\
-2 & Lower \\
-3 & Definitely lower \\
\hline
\end{tabular}




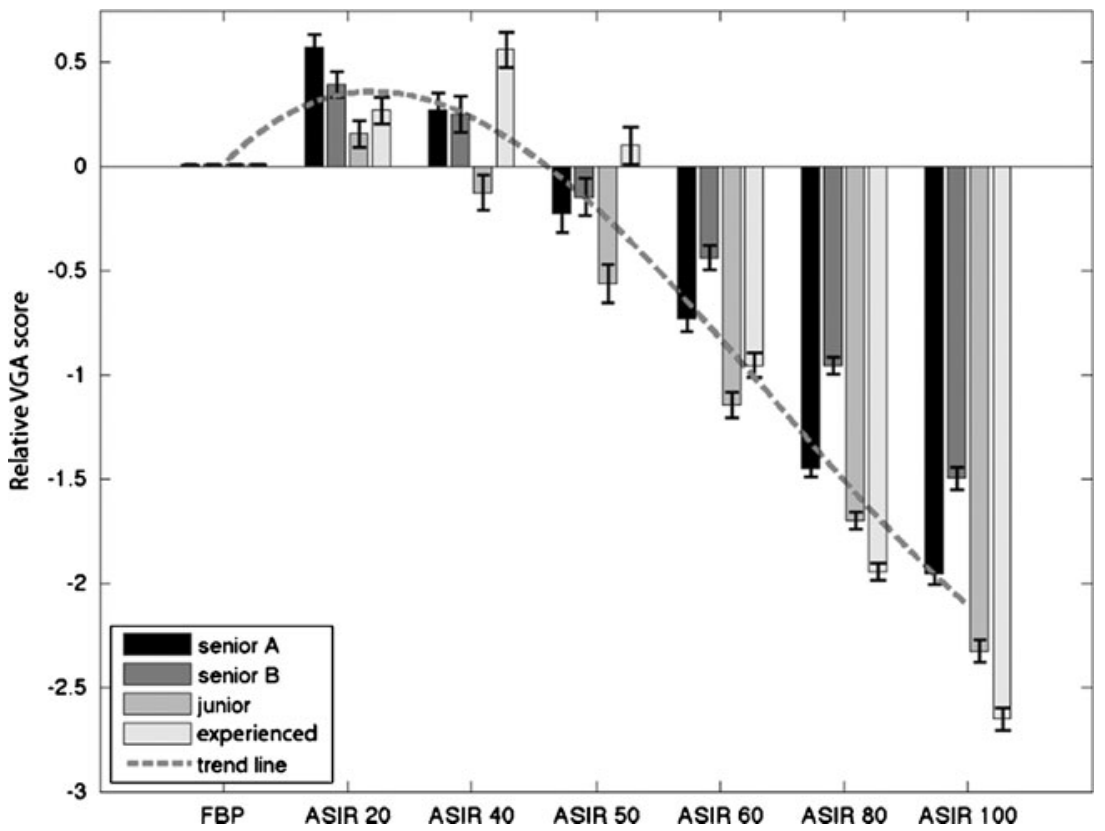

Fig. 3 Relative VGA scores of structure conspicuity as a function of ASIR percentage. The trend line corresponds to a spline line fitted across the average of the VGA scores. The highest structure

A statistically significant difference between ASIR reconstructions was obtained (repeated measures ANOVA, $p<0.001$, for all pair-wise comparisons). The optimum structure visibility was found with ASIR percentage of $20 \%$ and $40 \%$. The associated VGA scores were $0.35 \pm 0.05$ and $0.27 \pm 0.05$, respectively, corresponding to "equal" to "slightly better" visibility of the structures. With increasing ASIR percentages up to $100 \%$, the visibility steadily decreased. With $100 \%$ ASIR, the VGA score equalled $-2.15 \pm 0.05$ corresponding to a "lower" structure visibility. Significant differences were obtained for the intergroup (junior, experienced, senior) comparisons (repeated measures ANOVA, $p<$ 0.001, for all pair-wise comparisons) as shown in Fig. 3. However, responses from all radiologists clearly demonstrated that ASIR leads to a slight visibility improvement at percentages from $10 \%$ to $40 \%$ and a reduction in visibility at higher percentages due to variation in the image conspicuity.

For all the structures evaluated (Table 1), 20\% and 40\% ASIR yielded the highest structure visibility enhancement. While no significant differences were found (repeated measures ANOVA, $p=0.305$ ) within the group of cardiac structures reconstructed with the standard convolution kernel, there was a statistically significant difference in the scores (repeated measures ANOVA, $p<0.05$, pair-wise comparisons) between this latter group and the pulmonary interstitium structure (reconstructed with the lung kernel). Figure 4 illustrates the effects of the reconstruction kernel. The relative VGA scores of similar structures (distalities) and the pulmonary interstitium are shown as a function of conspicuity is between $20 \%$ and $40 \%$ ASIR whereas a steady reduction in structure conspicuity occurs from $50 \%$ to $100 \%$ ASIR

the ASIR percentage. The associated VGA scores were $0.35 \pm 0.11$ and $0.09 \pm 0.11$, respectively, at $20 \%$ ASIR.

To show the changes in the images introduced by ASIR when used with the standard convolution kernel, $40 \%$ and $100 \%$ ASIR were compared with FBP. To evaluate the differences between the images reconstructed using the two

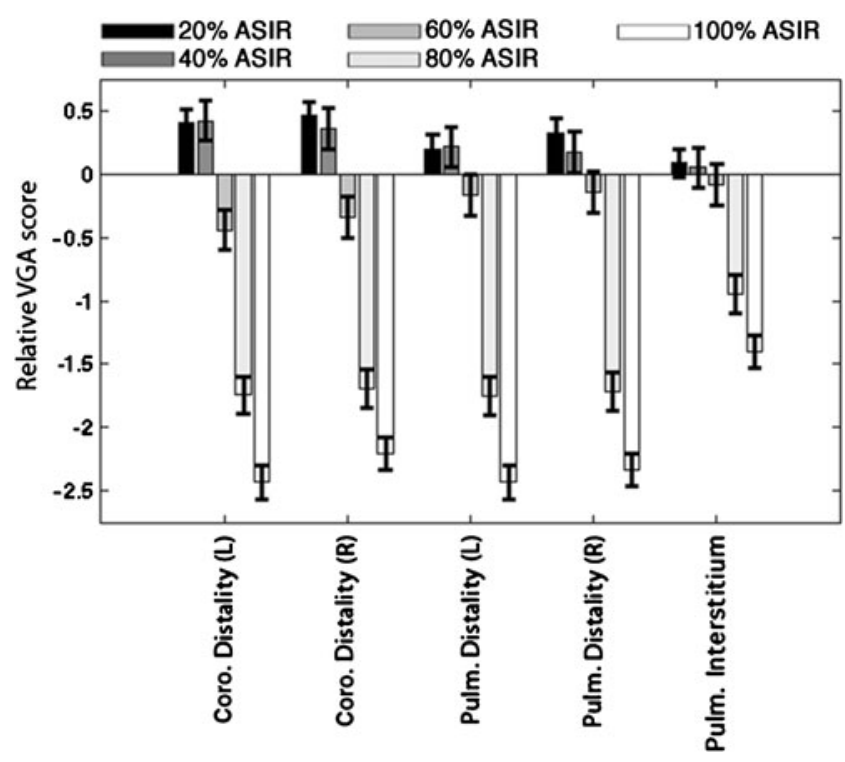

Fig. 4 Relative VGA scores of the coronary and pulmonary distalities as well as the pulmonary interstitium with increasing ASIR percentage. The image conspicuity of the structures reconstructed with the standard convolution kernel is homogeneous in contrast to the pulmonary interstitium reconstructed with the lung kernel, which is less affected by the ASIR percentage than the other cardiac structures 

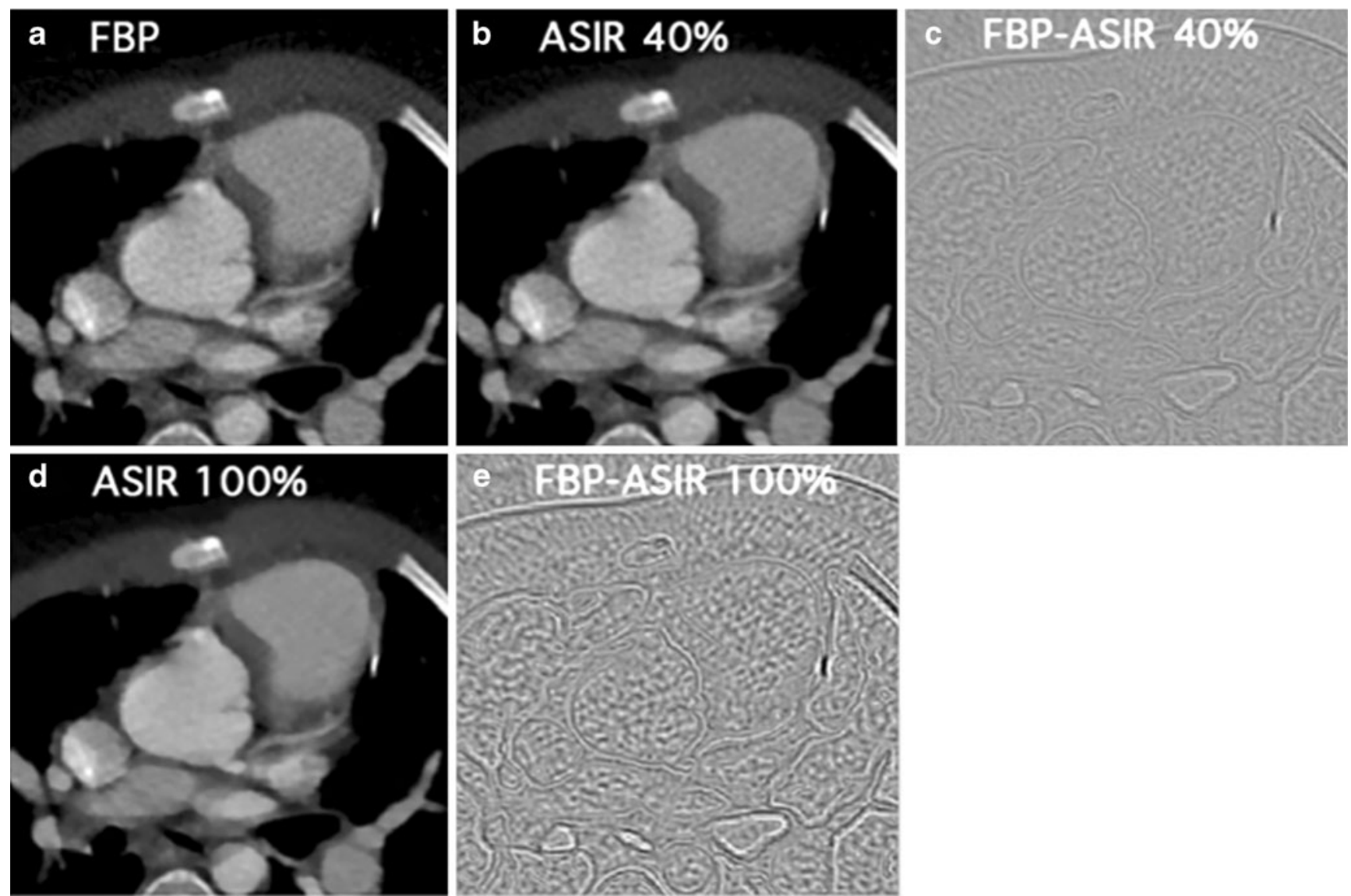

Fig. 5 Effects of $40 \%$ and $100 \%$ ASIR on the conspicuity of the cardiac structures in a 4-year-old boy when reconstructed with the standard convolution kernel and the following reconstruction methods: (a) FBP, (b) $40 \%$ ASIR, (e) $100 \%$ ASIR. c, e Pixel-to-pixel image subtractions of $(\mathbf{a}-\mathbf{b})$ and $(\mathbf{a}-\mathbf{d})$, respectively. The acquisition was

methods, the subtractions of the FBP-based and iterativebased images are presented in Fig. 5c, e. While 40\% ASIR decreased the image noise and slightly modified the borders of the structures, 100\% ASIR (Fig. 5e) almost totally removed image noise and clearly modified the structure outlines, especially between tissues and air. The strongest differences between FBP and iterative reconstructions were obtained with structures presenting high attenuation variations, such as the ossified ribs and sternum.

\section{Effect of ASIR on low- and high-contrast structures}

In a previous study [17], the behaviour of ASIR using the quality control phantom was shown to lead to a steady noise reduction as a function of the ASIR percentage. Figure 6 shows the effects of the two extreme conditions (FBP and 100\% ASIR) on simple low- and high-contrast structures of the phantoms. Acquisitions were performed at 8 mGy and 12 mGy for the paediatric and Catphan phantoms, respectively. In spite of a drastic noise reduction (from $12.7 \pm 0.3 \mathrm{HU}$ to $6.6 \pm 0.3 \mathrm{HU}$ ), no major improve- performed at $80 \mathrm{kVp}$ and $7.91 \mathrm{mGy}$. The window parameters were not modified between images (a, b and $\mathbf{d}$ ), nor between images (c and $\mathbf{e}$ ), ( $-50 / 800$ and $-125 / 80$, respectively). Compared with $40 \%$ ASIR, $100 \%$ ASIR yields a more pronounced noise reduction and structure outline modifications

ment in the visibility of the low-contrast structure was observed (Fig. 6a, b). Indeed, the low-contrast cylinder is not visible in Fig. 6c. On the other hand, the visibility of the resolution bar patterns was slightly improved as shown in Fig. 6d, e. The central bar patterns, which were not visible with the FBP method, were discernible with $100 \%$ ASIR. The background SDs were $22.2 \pm 0.3 \mathrm{HU}$ and $11.1 \pm 0.3 \mathrm{HU}$, respectively.

\section{Potential for dose reduction}

Since a slight improvement in image quality was observed with $20 \%$ and $40 \%$ ASIR (Fig. 3), estimating the potential dose reduction while maintaining image noise constant was important. Figure 7 illustrates the behaviour of the noise amplitude as a function of the $\mathrm{CTDI}_{\mathrm{vol}}$ when the FBP method and ASIR at different percentages were used. As the tube voltage can be adapted to patient size, measurements performed at $80 \mathrm{kVp}$ and $100 \mathrm{kVp}$ are given. As shown in both graphs of Fig. 7, an important reduction of image noise occurred with increasing ASIR percentage. 


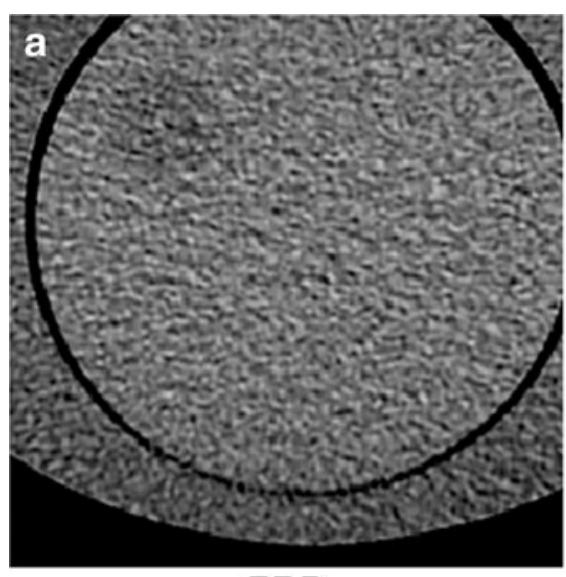

FBP

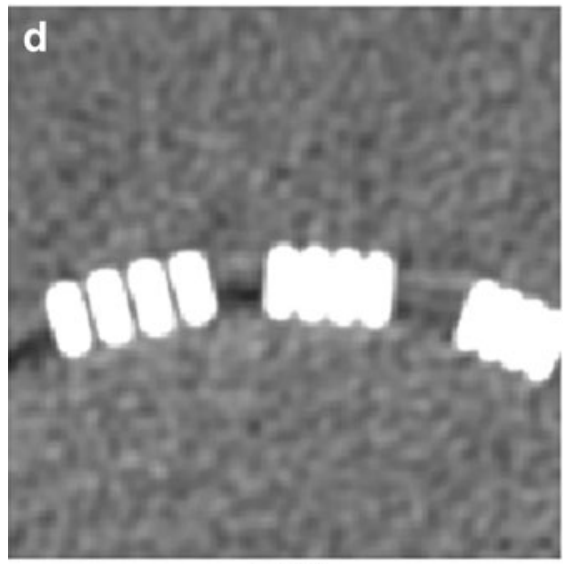

FBP

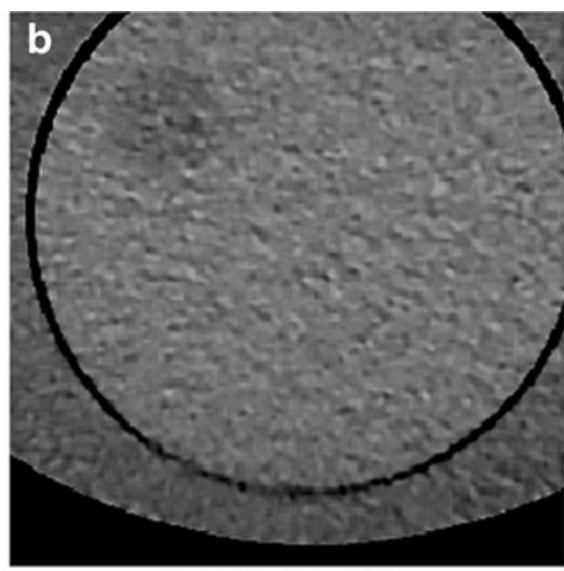

ASIR $100 \%$

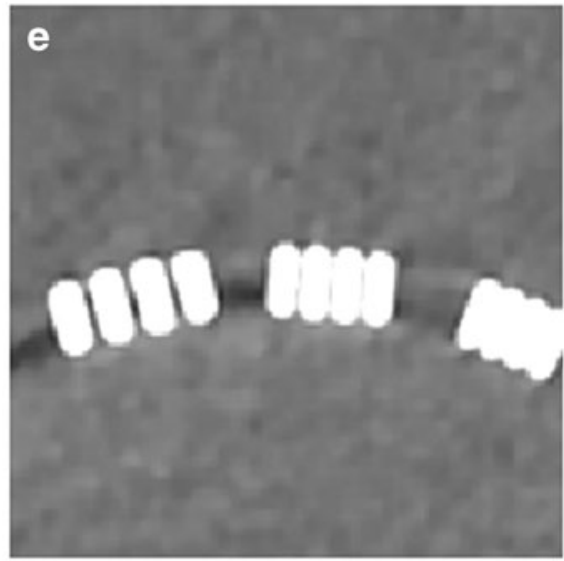

ASIR $100 \%$

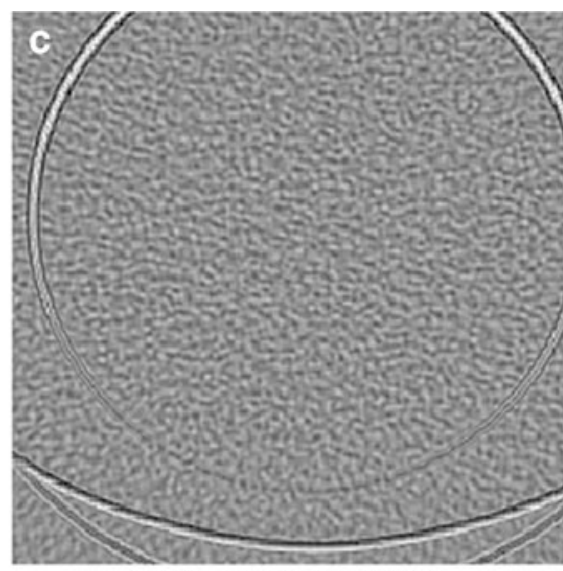

FBP-ASIR100\%

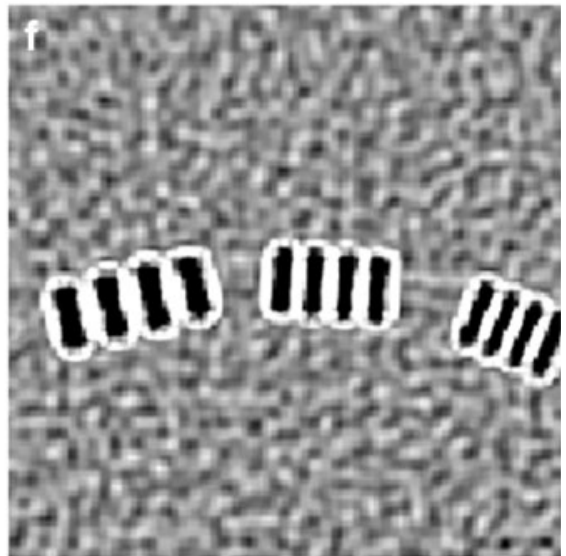

FBP-ASIR 100\%
Fig. 6 Effects of $100 \%$ ASIR on the visibility of the low-contrast cylinder (paediatric phantom) and on the resolution bar patterns (CTP515 module of the Catphan 600 phantom) when reconstructed with the standard reconstruction kernel. $(\mathbf{a}-\mathbf{c})$ Images obtained from the paediatric phantom (a) with FBP, (b) with $100 \%$ ASIR, and (c) by pixel-to-pixel image subtraction of $(\mathbf{a}-\mathbf{b})$. $\mathbf{d}-\mathbf{f}$ Images obtained from

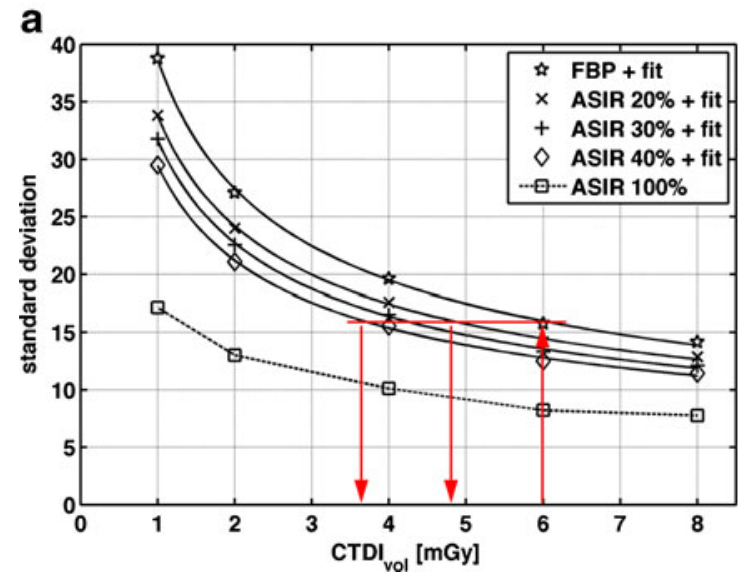

Fig. $7 \mathrm{SD}$ as a function of $\mathrm{CTDI}_{\mathrm{vol}}$ for different ASIR percentages and two tube voltages: $80 \mathrm{kVp} \mathrm{(a)} \mathrm{and} 100 \mathrm{kVp}$ (b). Acquisitions were performed with the paediatric protocol on the paediatric phantom. A SD of $15.5 \mathrm{HU}$ is measured at $6 \mathrm{mGy}$ with the FBP method while the same the Catphan phantom (d) with FBP, (e) with $100 \%$ ASIR, and (f) by pixel-to-pixel image subtraction of $(\mathbf{e}-\mathbf{f})$. The white areas correspond to the biggest differences between ( $\mathbf{a}$ and $\mathbf{d}$ ) and between (b and $\mathbf{e}$ ), while black areas indicate no differences. While no significant contrast enhancement is visible (c), better resolution is obtained (f) with $100 \%$ ASIR

b

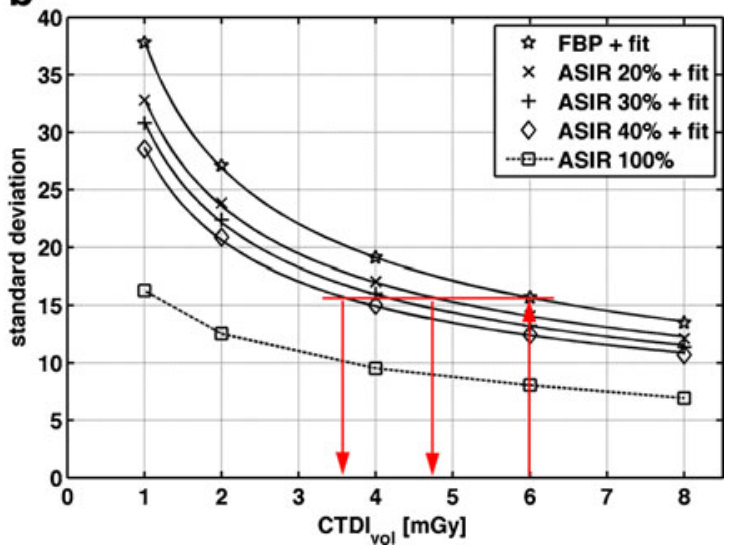

noise value is related to a $\mathrm{CTDI}_{\mathrm{vol}}=3.8 \mathrm{mGy}$ and $3.7 \mathrm{mGy}$ with $40 \%$ ASIR when acquired at $80 \mathrm{kVp}$ and $100 \mathrm{kVp}$, respectively. A fit was added for reconstructions following the $\left(1 / \mathrm{CTDI}_{\mathrm{vol}}\right)^{1 / 2}$ relationship 
Since the CT dose in a child ages $2-3$ years is around $6 \mathrm{mGy}$ when the NI is fixed at $15.5 \mathrm{HU}$, we chose this value as the reference value. The figure shows that a theoretical dose reduction associated with a $\mathrm{CTDI}_{\mathrm{vol}}$ equal to $3.8 \mathrm{mGy}$ and $3.7 \mathrm{mGy}$ would be possible with $40 \%$ ASIR when a child is scanned at $80 \mathrm{kVp}$ and $100 \mathrm{kVp}$, respectively. These values would lead to corresponding dose reductions of $36 \pm 2 \%$ and $38 \pm 2 \%$. With $20 \%$ ASIR, the dose reductions are weaker and are $18 \pm 2 \%$ and $20 \pm 2 \%$ for $80 \mathrm{kVp}$ and $100 \mathrm{kVp}$, respectively.

\section{Discussion}

The purpose of this study was to evaluate the benefit of different ASIR percentages in enhancing the diagnostic quality of paediatric cardiac CT scans. Based on the results of the VGA method, the best enhancement of structure visibility in paediatric cardiac scans was obtained with $20 \%$ and $40 \%$ ASIR (Fig. 3). With these reconstruction approaches, the structure visibility on images reconstructed with the standard convolution kernel was considered as "equal" to or "slightly better" than (VGA score $=0.35 \pm$ 0.05 ) visibility obtained from images reconstructed with the conventional FBP method. Radiologists reported that the images reconstructed with $20 \%$ and $40 \%$ ASIR were less noisy and had a better overall conspicuity than those reconstructed with FBP.

To assess image quality, several methods are generally used. A state-of-the-art and one of the most reliable methods is receiver operating characteristics (ROC) analysis [18]. Although this method is able to measure the ability of an observer to correctly detect a signal in a clinical image, its use is relatively time-consuming and complex. Moreover, a ROC study requires the addition a signal mimicking a pathology to the images that is not directly possible with commercial CT scanners. In contrast, purely physical methods based on metrics, such as the modulation transfer function, the noise power spectra or the detector quantum efficiency, are well adapted for describing the quality of the equipment itself [18], but clinical performance cannot be predicted from determinations of these parameters only since they are not linked with a diagnostic task [20, 21]. A good compromise for evaluating the diagnostic image quality is the VGA method. Such analysis can be used when a set of anatomical criteria related to a clinical examination is previously known. By comparing different images of the same anatomical part of the body, the clinical image quality can be evaluated in a systematic and scientific manner [22].

In the design of our VGA study, we first established a set of anatomical criteria that paediatric radiologists implicitly check when they perform paediatric cardiac examinations.
From among this set of criteria, we retained the 19 structures judged as the most relevant (Table 1). Almost all the chosen structures behaved in a similar manner in relation to the ASIR percentage used in the image reconstruction. This was rather unexpected a priori. However, an explanation of this similar behaviour can be found in the nature of these structures, which are all soft tissues in opposition to bone structures.

Surprisingly, it appears that the benefit of ASIR is significantly dependent on the convolution kernel (Fig. 4). The enhancement provided by $20 \%$ and $40 \%$ ASIR is reduced when using the lung convolution kernel in comparison to the standard reconstruction kernel. Indeed, the visibility of the lung interstitium showed only marginal improvements (VGA score $0.09 \pm 0.11$ ) and was evaluated as "equal" when compared to the reference image.

Our results confirm the fact that, as already mentioned by several authors [6, 7,9], 100\% ASIR yields a significantly "lower" structure visibility than standard FBP. Radiologists unanimously reported a loss in the conspicuity of the structures due to the "aggressive" suppression of image noise. Besides the severe noise-free effect present on all 100\% ASIR images, anatomical details of organs appeared to be affected by the formation of pixel clusters (Fig. 2). Also, structure outlines were reported to be less sharp than the original ones. However, although $100 \%$ ASIR was associated with a VGA score indicating "lower structure visibility," and thus lower image conspicuity, it was not possible to accurately determine if this reconstruction could affect diagnostic confidence.

Our statistical analysis showed significant scoring variations among observers as shown in Fig. 3. These variations may have three sources. The first is the experience of the radiologist with cardiac examinations. Statistically significant differences were observed among radiologists while no significant differences were observed between the two senior radiologists (coming from two different hospitals). The second source may be the interpretation of the relative VGA scale [23]. In spite of accurate explanations before starting scoring examinations, differences in the perception of the scale steps led to significant score variations. This effect is particularly visible with $100 \%$ ASIR, where scoring varied between $-1.49 \pm 0.08$ and -2.65 \pm 0.08 . Moreover, the slight modifications in structure visibility produced by the different ASIR percentages could also be responsible for this difference.

To investigate whether $100 \%$ ASIR improved the perceived contrast of structures, images of a low-contrast phantom were reconstructed with $100 \%$ ASIR (Fig. 6). It was shown that ASIR removes image noise mainly by reducing the variations in pixel values between neighbouring pixels without amplifying the perceived contrast. The analysis of the phantom containing high-contrast objects 
showed that $100 \%$ ASIR reduces image noise but, in addition, reinforces the edge transitions between the structure and its background, which improves the visibility of highcontrast patterns. In the framework of cardiac paediatric examinations, opacified structures could benefit from such an effect.

The main purpose of employing ASIR is to reduce patient dose, but before focusing on this aspect it is important to first study the impact of this new reconstruction method at a given dose level. To have an idea of the potential dose reduction, we used phantom images to investigate the noise reduction provided by ASIR at various percentages and then estimated the dose reduction with $20 \%$ and $40 \%$ ASIR, the optimal levels obtained during this study. With data acquisition corresponding to a child ages $2-3$ years and for a standard noise image $(15.5 \mathrm{HU})$ and an initial $\mathrm{CTDI}_{\mathrm{vol}}$ of $6 \mathrm{mGy}$, a dose reduction of $18 \%$ with $20 \%$ ASIR and $36 \%$ with $40 \%$ ASIR was obtained. In practice with our paediatric protocol, such dose reductions can be reached by setting the NI to $17.5 \mathrm{HU}$ and $20.5 \mathrm{HU}$, respectively (Fig. 7).

These results are in good agreement with those of Hara et al., where dose reductions of $32-65 \%$ were obtained for abdomen CT protocols in adults when using ASIR 40\% [7]. Recently, Leipsic et al. have also shown that in adult CT angiography $40 \%$ to $60 \%$ ASIR significantly improves image quality and the proportion of interpretable segments [9]. In adult chest CT examinations, $30 \%$ ASIR was chosen because it allows image quality similar to that of FBP and affords the opportunity for dose reduction [24]. Therefore, in addition to the numerous strategies already proposed for reducing doses in CT [3-5], especially in cardiac CT examinations [25] and in paediatric CT [4, 26-31], new advanced reconstruction methods, including ASIR, are promising and should be considered.

A limitation of this study was the relatively small number of patients involved. Due to the relatively large number of anatomical structures considered, the analysis was already quite time-consuming (about 4 hours per radiologist for the whole study) and it was difficult to increase the size of the cohort. The results obtained indicate that we could have included more children and reduced the number of quality criteria. However, in spite of this, we obtained clear trends with statistical significance. Another restriction was the impossibility of comparing the FBP method with itself to assess the intraobserver variability. This was due to the restriction of our picture archiving and communication systems, which did not allow the duplication of the scans. However, from $20 \%$ up to $100 \%$ ASIR, ASIR-based images were distinguishable from FBP-based images and it is very unlikely that important intraobserver variability could have occurred.

\section{Conclusion}

Based on a VGA carried out by four paediatric radiologists, we conclude that reconstruction including $20 \%$ to $40 \%$ ASIR slightly improved the conspicuity of various paediatric cardiac structures in newborns and children with respect to conventional reconstruction (FBP) alone. However, 100\% ASIR was associated with a decrease in the VGA score due to its aggressive noise reduction, and with a blurring of anatomical structure outlines. The statistical analysis showed that the image conspicuity of all structures reconstructed with the standard convolution kernel was homogeneous regardless of the ASIR percentage, whereas a difference in image conspicuity was observed between cardiac structures reconstructed with the standard convolution kernel and the lung structure reconstructed with the lung kernels.

Dose reductions of about $18 \%$ and $36 \%$ were obtained with a paediatric phantom with $20 \%$ and $40 \%$ ASIR, respectively, when using a weighted-based paediatric cardiac CT protocol. Therefore, we think that iterative algorithms are promising for dose reduction even in lowvoltage, low-current scans in children and young adults.

Acknowledgments We would like to thank GE Healthcare and especially Christophe Argaud and Paul Ayestaran for their assistance in image reconstruction; Christel Elandoy and Martine Bernasconi for their help with image acquisitions and for providing the machine time required to perform the study, and Leonor Alamo, Elodie Senggen and Rafael Duran for their participation in this study.

This research was supported by a Swiss National Science Foundation grant [no. 320030-120382].

\section{References}

1. Pierce DA, Shimizu Y, Preston DL et al (1996) Studies of the mortality of atomic bomb survivors. Report 12, Part I. Cancer: 1950-1990. Radiat Res 146:1-27

2. Brenner DJ, Hall EJ (2007) Computed tomography: an increasing source of radiation exposure. N Engl J Med 357:2277-2284

3. McCollough CH, Bruesewitz MR, Kolfer JM (2006) CT dose reduction and dose management tools: overview of available options. Radiographics 26:503-512

4. Coursey C, Frush DP, Yoshizumi T et al (2008) Pediatric chest MDCT using tube current modulation: effect on radiation dose with breast shielding. AJR 190:W54-W61

5. Gunn MLD, Kohr JR (2009) State of the art: technologies for computed tomography dose reduction. Emerg Radiol 17:209-218

6. Silva AC, Lawder HJ, Hara A et al (2010) Innovations in CT dose reduction strategy: application of the adaptive statistical iterative reconstruction algorithm. AJR 194:191-199

7. Hara AK, Paden RG, Silva AC et al (2009) Iterative reconstruction technique for reducing body radiation dose at CT: feasibility study. AJR 193:764-771

8. Marin D, Nelson RC, Schindera ST et al (2010) Low-tubevoltage, high-tube-current multidetector abdominal CT: improved image quality and decreased radiation dose with adaptive statistical iterative reconstruction algorithm - initial clinical experience. Radiology 254:145-153 
9. Leipsic J, LaBounty TM, Heilbron B et al (2010) Adaptive statistical iterative reconstruction: assessment of image noise and image quality in coronary CT angiography. AJR 195:649-654

10. Thibault JB, Sauer KD, Bouman CA et al (2007) A threedimensional statistical approach to improved image quality for multislice helical CT. Med Phys 34:4526-4544

11. Hsieh J (2009) Computed tomography: principles, design, artifacts, and recent advances, 2nd edn. SPIE, Bellingham, WA

12. Swiss Federal Office for Public Health (2010) Notice R-06-06. Bern, Switzerland. http://www.bag.admin.ch/themen/strahlung/ 02839/index.html?lang=fr. Accessed 17 May 2011

13. Verdun FR, Gutierrez D, Vader JP et al (2008) CT radiation dose in children: a survey to establish age-based diagnostic reference levels in Switzerland. Eur Radiol 18:1980-1986

14. International Electrotechnical Committee (1994) Medical diagnostic X-ray equipment - radiation conditions for use in the determination of characteristics. Standard IEC 61267, Geneva

15. International Electrotechnical Committee (1999) Medical diagnostic X-ray equipment - particular requirements for the safety of X-ray equipment for CT. Standard IEC 0601-2-44, Geneva

16. International Electrotechnical Committee (2002) Medical diagnostic $\mathrm{X}$-ray equipment - particular requirements for the safety of X-ray equipment for CT, 2nd edn. Standard IEC 60601-2-44, Geneva

17. Miéville FA, Ayestaran P, Argaud C et al (2010) Potential benefit of the CT adaptive statistical iterative reconstruction method for pediatric cardiac diagnosis. Proc SPIE 7622:76222D

18. Månson LG (2000) Methods for the evaluation of image quality: a review. Radiat Prot Dosim 90:89-99

19. European Commission (1999) EUR 16262: European guidelines on quality criteria for computed tomography. Office for Official Publications of the European Communities, Luxembourg

20. Båth M (2010) Evaluating imaging systems: practical applications. Radiat Prot Dosim 139:26-36
21. Sund P, Båth M, Kheddache S et al (2004) Comparison of visual grading analysis and determination of detective quantum efficiency for evaluating system performance in digital chest radiography. Eur Radiol 14:48-58

22. Tingberg A, Hermann C, Lanhede B et al (2000) Comparison of two methods for evaluation of the image quality of lumbar spine radiographs. Radiat Prot Dosim 90:165-168

23. Båth M, Månson LG (2007) Visual grading characteristics (VGC) analysis: a non-parametric rank-invariant statistical method for image quality evaluation. Br J Radiol 80:169-176

24. Leipsic J, Nguyen G, Brown J et al (2010) A prospective evaluation of dose reduction and image quality in chest CT using adaptive statistical iterative reconstruction. AJR 195:1095-1099

25. Paul JF, Abada HT (2007) Strategies for reduction of radiation dose in cardiac multislice CT. Eur Radiol 17:2028-2037

26. Li X, Samei E, Segars WP et al (2008) Patient-specific dose estimation for pediatric chest CT. Med Phys 35:5821-5828

27. Herzog C, Mulvihill DM, Nguyen SA et al (2008) Pediatric cardiovascular CT angiography: radiation dose reduction using automatic anatomic tube current modulation. AJR 190:12321240

28. Singh SS, Kalra MK, Moore MA et al (2009) Dose reduction and compliance with pediatric CT protocols adapted to patient size, clinical indication, and number of prior studies. Radiology 252:200-208

29. Paul JF, Abada HT, Sigal-Cinqualbre A (2004) Should lowkilovoltage chest CT protocols be the rule for pediatric patients? AJR 138:1172; author reply 1172

30. Vock P (2005) CT dose reduction in children. Eur Radiol 15:2330-2340

31. Strauss KJ, Goske MJ, Kaste SC et al (2010) Image gently: Ten steps you can take to optimize image quality and lower CT dose for pediatric patients. AJR 194:868-873 